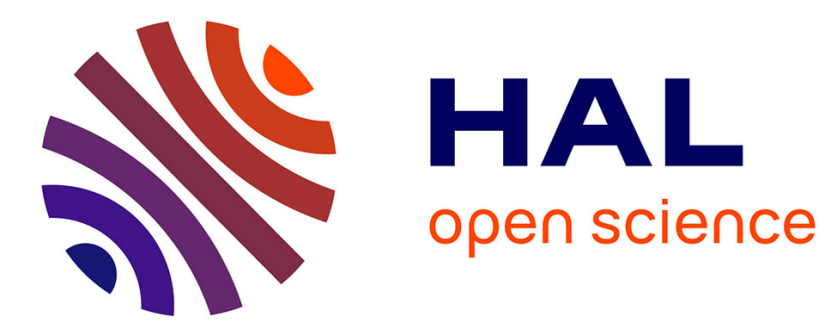

\title{
Les jeunes Roumaines sont des garçons comme les autres
}

Arthur Vuattoux

\section{To cite this version:}

Arthur Vuattoux. Les jeunes Roumaines sont des garçons comme les autres. Plein Droit, 2015, 104

(1), 10.3917/pld.104.0027 . halshs-01849053

\section{HAL Id: halshs-01849053 \\ https://shs.hal.science/halshs-01849053}

Submitted on 25 May 2019

HAL is a multi-disciplinary open access archive for the deposit and dissemination of scientific research documents, whether they are published or not. The documents may come from teaching and research institutions in France or abroad, or from public or private research centers.
L'archive ouverte pluridisciplinaire HAL, est destinée au dépôt et à la diffusion de documents scientifiques de niveau recherche, publiés ou non, émanant des établissements d'enseignement et de recherche français ou étrangers, des laboratoires publics ou privés. 


\section{Les jeunes Roumaines sont des garçons comme les autres.}

Arthur Vuattoux, Université Paris 13, Institut de recherche interdisciplinaire sur les enjeux sociaux (IRIS), vuattoux@univ-paris13.fr

Lors d'une enquête sociologique menée en Île-de-France afin d'éclairer qualitativement les biais de genre présents dans la chaîne judiciaire concernant les mineurs (justice civile et justice pénale), le cas des "jeunes filles roumaines» - repérées et nommées ainsi par les professionnels de la justice des mineurs - est apparu comme un cas limite. Échappant aux schémas genrés qui président habituellement à la justice des mineurs (au sens où les adolescentes délinquantes évitent généralement la prison au profit de prises en charge para-pénales, médico-sociales notamment), les "jeunes filles roumaines" sont largement pénalisées, et connaissent la prison de manière très fréquente pour de simples vols. On peut avancer qu'elles subissent un traitement judiciaire d'exception, révélateur des discriminations multiples vécues par les Roms sur le territoire français.

La recherche présentée ici a été mise en œuvre à partir d'un territoire (l'Île-de-France), d'une institution (la justice des mineurs) et d'un public (les adolescents Roms, et plus spécifiquement les adolescentes Roms) ${ }^{\mathrm{i}}$. Ce regard situé sur les discriminations a pour objectif d'informer sur la manière dont l'État fait face à une entité minoritaire qu'« il » (l’État, via un ensemble d'acteurs, de discours et de politiques publiques) a contribué à minoriser. Comment les institutions, à l'échelle locale, peuvent-elles résoudre (ou non) la tension existant a priori entre, d'une part, une institution judiciaire présentée comme la garante d'un traitement équitable des personnes, et, d'autre part, un contexte d'exclusion sociale et politique d'une population précisément amenée à côtoyer la justice du fait du contrôle policier massif qu'elle subit au quotidien? La justice rééquilibre-t-elle les discriminations subies par les populations Roms dans la société, quand bien même celles-ci se présenteraient à elles sous la figure de l'adolescence déviante, ou entérine-t-elle au contraire les discriminations en les reproduisant entre les murs des tribunaux, puis, par extension, entre ceux des prisons ? Pour le dire simplement, cette recherche vise à analyser un cas limite au sein d'une étude plus vaste de la justice des mineurs d'aujourd'huii ${ }^{\mathrm{i}}$ : il s'agit de questionner une situation dans laquelle les ressorts habituels de cette justice (souvent présentée comme bienveillante, voire laxiste, du fait de la priorité à l'éducation sur la répression qui en constitue le fondement ${ }^{\mathrm{iii}}$ ) ne semblent plus fonctionner.

\section{La mise au jour d'un « circuit particulier » au sein de la justice des mineurs}

Essentiellement perçus à travers les dimensions répressives de l'action publique, notamment à travers les expulsions de bidonvilles, les Roms d'Île-de-France vivent dans cette région le même type de discriminations que celles qu'ils et elles vivent partout en Europe ${ }^{\text {iv }}$. Cependant, l'enquête menée ici dans deux tribunaux franciliens permet de percevoir des spécificités locales notables. Les enjeux locaux du traitement judiciaire des adolescents Roms ont été étudiés à partir de deux juridictions, Paris et Créteil. Je ne présenterai dans cet article que la situation parisienne, où les mineurs Roms représentent plus de la moitié de l'activité du Tribunal pour enfants.

Si ces mineurs sont soumis, en tant que mineurs isolés étrangers (MIE), aux mêmes procédures que les «autres» mineurs (les mineurs «domiciliés »), le traitement qui leur est réservé dans les faits révèle l'existence d'un traitement particulier. Une éducatrice d'un service de la Protection judiciaire de la jeunesse (PJJ) d'une juridiction attenante à celle de Paris m'indiquera sa stupéfaction face aux premiers mineurs roumains déférés dont elle a suivi la situation au tribunal :

«Plusieurs fois, par exemple, je suis intervenue pour des enfants roumains de moins de 13 ans qu'on mettait en cellule... Alors maintenant, les policiers font un peu plus attention, mais je leur rappelais que c'était pas avant 13 ans les cellules... Et on me répondait, "bon, mais ça va, c'est des roumains...". Et même au niveau du tribunal il y a un traitement particulier (...). Dès le départ, on nous annonce qu'il y a des "roumains", on sait que ça va être un circuit particulier, que systématiquement ça va être un jugement à délai rapproché... Et ça ça me choquait beaucoup au début: j'avais l'impression qu'il y avait une justice pour tout le monde et une justice pour les roumains... Et j'ai l'impression que ce n'est pas du tout questionné, encore maintenant, même par des gens qu'on estime beaucoup, qu'on sait être au-delà des considérations racistes ou autres... C'est dans la tête de beaucoup une catégorie à part... » (Anne Séris ${ }^{v}$, éducatrice à la PJJ, Île-deFrance).

La franchise de cette enquêtée, qui ne veut pas parler de racisme mais qui évoque un traitement clairement défavorable aux adolescents catégorisés comme « roumains » en vertu même de leur origine, rejoint mon impression générale face aux pratiques de l'institution. La lecture des dossiers judiciaires, et plus spécifiquement de ceux des filles, confirmera d'ailleurs l'idée d'un « circuit particulier » tel que l'évoque cette éducatrice : la prison, comme réponse courante à leurs délits, fait figure d'exception dans le fonctionnement routinier de la justice des mineurs, qui tend habituellement à 
orienter les filles vers d'autres types de prise en charge (sociales et sanitaires notamment) ${ }^{\mathrm{vi}}$. J'ai donc fait le choix de centrer l'analyse sur ces seules adolescentes ou « jeunes filles roumaines » ${ }^{\text {vii }}$, choix qui ne doit bien-sûr par faire oublier le traitement judiciaire différentiel que subissent, dans une moindre mesure, les garçons dits « roumains ».

\section{Les « jeunes filles roumaines » sont des garçons comme les autres}

En quoi la situation des «jeunes filles roumaines » diffère t-elle de celle des autres mineurs pris en charge par la justice ? Au vu des dossiers étudiés au tribunal de Paris et dans d'autres juridictions d'Île-de-France, on remarque que la distance n'est pas la même entre le traitement judiciaire des garçons Roms et non-Roms, et entre celui des filles Roms et non-Roms. Les garçons Roms, dont les actes de délinquance (essentiellement des actes de petite délinquance : vol, recel, escroquerie) sont fortement réprimés, ne sont certes pas tout à fait traités «comme» les autres garçons, au sens où ils ne sont pas orientés vers le même type de prise en charge, mais la réponse pénale qui leur est fréquemment apportée, la privation de liberté (souvent après des premières mesures de liberté surveillée préjudicielle), est une réponse courante parmi l'ensemble des délinquants garçons (même si elle semble intervenir de manière plus fréquente chez les garçons Roms). Les filles en revanche, catégorisées par les acteurs rencontrés sous le vocable de «jeunes filles roumaines », subissent un traitement qui diffère très nettement de celui des autres jeunes filles et qui les rapproche fortement du traitement habituellement réservé aux garçons.

$\mathrm{Au}$ tribunal de Paris, on assiste à une importante mobilisation institutionnelle autour des situations des adolescents roumains, filles comme garçons. Le volet ethnographique de cette recherche ${ }^{\text {viii }}$ a été concentré sur l'échelon judiciaire, et plus particulièrement sur les services de la PJJ, centraux dans la prise en charge des mineurs au pénal. Le Service territorial éducatif de milieu ouvert de Paris (STEMO Paris-Centre, PJJ), regroupant à la fois un service de milieu ouvert (nommé Unité éducative de milieu ouvert Lafayette) et un service d'investigation (l'UEAT), a initié la mise en place d'un dispositif spécifiquement tourné vers les adolescents roumains. Ce dispositif se caractérise notamment par la présence, dans les locaux du STEMO, d'un éducateur roumanophone et d'une assistante sociale elle-aussi roumanophone.

Le dispositif consiste principalement en la mise en place de « rendez-vous éducatifs » au STEMO, en parallèle de la procédure judiciaire. Ainsi, lorsqu'un adolescent ou une adolescente est mis en examen, le juge lui notifie un rendezvous avec le service éducatif. Lors de ces rendez-vous, qui ne sont pas systématiquement honorés par les jeunes ${ }^{\text {ix }}$, ils et elles peuvent rencontrer l'éducateur ou l'assistante sociale roumanophones, qui travaillent à leur expliquer la procédure, à les aiguiller vers d'autres structures (associatives notamment, malgré le manque de place dans ces structures) ou encore à leur fournir une aide immédiate (tickets alimentaires, aide au transport, accompagnement vers des professionnels du soin - dentistes notamment).

Relevant d'une volonté d'inclusion des adolescents Roms via une adaptation du dispositif de droit commun, au sens où l'institution fait le choix de s'adapter à un nouveau «public», les efforts mis en œuvre à Paris semblent cependant achopper sur la réalité du traitement judiciaire, qui continue en parallèle de faire son œuvre. En effet, malgré cette volonté institutionnelle d'adaptation aux situations des adolescents Roms, et malgré l'élaboration par les professionnels du STEMO d'une réflexion approfondie sur ces adolescents et leurs parcours, la réalité du traitement pénal semble peu affectée.

À Paris, les adolescentes roumaines connaissent la prison de manière très fréquente, y compris pour des vols simples et autres délits de même catégorie. D'après des données difficiles à recouper (essentiellement issues de rapports d'activités que j'ai pu me procurer et du recoupement de propos tenus à ce sujet par les enquêtés), il semblerait qu'il y ait environ 100 adolescents Roms incarcérés chaque année (dans les dernières 3 années tout au moins) sur 200 mineurs différents (et environ 1000 défèrements $^{\mathrm{x}}$ ). Cela aboutit à un pourcentage très élevé d'adolescents Roms incarcérés, puisqu'environ $50 \%$ d'entre elles et eux connaîtraient la prison chaque année. Les filles représenteraient près de $50 \%$ de l'ensemble de ces mineurs. Souvent, des mesures de sursis s'accumulent et finissent, comme le disent les acteurs, par «tomber», amenant des jeunes filles à Fleury-Mérogis pour des durées parfois importantes et dans des conditions souvent difficiles, comme l'expose Elena Terescu, travailleuse sociale à Paris :

« Il y a une fille elle a accouché en prison, c'est la deuxième fille qui accouche en prison, elle était assez déprimée parce qu'elle savait qu'elle devait rester pour un an... Il y a aussi le fait que tu as une peine de 6 mois, puis tu as l'éducateur qui vient et qui te dit "ta sortie, c'est telle date", mais à "telle date", il y a une autre peine qui tombe... Elles pensent qu'elles sortent et en fait elles ne sortent pas... Donc si elles se retrouvent avec deux, trois dossiers de 2 mois ou 3 mois, elles vont rester pour un bout de temps. Et à chaque fois quelqu'un leur dit: "là c'est la sortie", et elles ne savent pas vraiment..

L'enquêteur : Et ça, ce sont des peines courantes, 2 mois, 3 mois?

Elena Terescu: Oui, parce que dernièrement, ils [les jeunes roumains] ont commencé à ne pas se présenter au jugement, et les peines s'accumulent, et quand ils se font chopper, il y a des peines de 6 mois, 9 mois... Il y a une fille ce mois-ci, elle est très déprimée, car elle a eu je pense 4 mois, et elle se demande pourquoi elle a eu 4 mois et l'autre 1 mois... Ben c'est parce que son dossier est un peu plus long que celui de l'autre... Un mois ça va, deux mois ça passe encore, mais trois mois, quatre mois, la dépression elle est garantie... »(Entretien avec Elena Terescu, Paris, janvier 2014). 
L'entretien réalisé avec un éducateur spécialisé de la juridiction, Guillaume Martin apporte une confirmation à ce constat d'un traitement sévère des adolescentes Roms, par opposition au traitement plutôt favorable réservé dans l'ensemble aux mineurs « domiciliés » (c'est-à-dire aux mineurs possédant une adresse fixe identifiée par la justice) :

«Guillaume Martin: On a des jeunes [roumains], au bout du premier jugement ils ont déjà une peine de prison. Avant, il fallait attendre 5, 6, 7 dossiers avant qu'il y ait une peine de prison... Là maintenant, c'est beaucoup plus rapide (...) On sent bien que par rapport aux autres mineurs, c'est un peu plus ferme... C'est rare de voir un mineur domicilié partir en détention... On a des exemples ici où il faut attendre $15 / 20$ défèrements avant qu'il y ait la première peine de prison...

L'enquêteur: C'est d'autant plus étonnant pour les filles non ? Puisque pour elles, la détention est beaucoup plus rare en général?

Guillaume Martin: Oui ! Ça c'est sûr... Et quand on va à Fleury, c'est principalement des roumaines, des personnes d'ex-Yougoslavie... En détention, les surveillantes elles ne sont pas mécontentes, car ça se passe très bien. C'est des jeunes respectueuses du cadre et des personnes. Une mineure qui part en prison, si elle est domiciliée, en général c'est beaucoup plus tendu. Quand on a des filles [sous-entendu : domiciliées] qui passent ici, elles sont beaucoup plus violentes. Les roumaines, c'est des gentilles à côté. Ça créé des incompréhensions. Des fois, je me dis, quand on voit des gamins qui passent ici pour des affaires de viol et qui ressortent avec un CJ [Contrôle judiciaire], et que nos gamins roumains, avec une seule condamnation, vont déjà en prison... On se dit il y a quand même deux poids deux mesures quoi...!» (Entretien avec Guillaume Martin, Paris, janvier 2014).

Les propos de cet éducateur traduisent bien le traitement discriminant vécu par les adolescentes Roms, y compris dans le lapsus qu'il manifeste en opposant « les filles » et « les roumaines », révélant ainsi une ségrégation interne au groupe des filles confrontées à la justice.

D'une part, il y a « les domiciliées », ces jeunes filles des quartiers populaires, parfois issues des classes moyennes ou aisées (situations relativement fréquentes à Paris, même si la majorité des mineures demeure issue des classes populaires), qui agissent contre la loi d'une manière conventionnelle et interprétable à travers des scripts de genre, de classe et de race eux-mêmes conventionnels. Qu'elles soient blanches ou non, issues de familles pauvres ou non, des scripts existent qui permettent d'apporter des clés à leur déviance : les déviances adolescentes féminines "types » prennent souvent la forme de conflits intra-familiaux, de violence en institution (foyers notamment) ou encore de vols à l'étalage.

D'autre part, à l'opposé de ces figures conventionnelles de la déviance adolescente, il y a les « jeunes filles roumaines », qu'on ne rattache à aucune des figures connues de l'adolescence déviante telles que la justice des mineurs a coutume de les appréhender (via une médiation avec la famille, ici absente, ou via l'institution scolaire et socio-sanitaire). Présentées comme " inéducables », « sans attache » ou tout simplement rattachées à l'âge adulte du fait de leur conjugalité ou de leur sexualité considérées comme «précoces», les «jeunes filles roumaines » font l'objet d'un traitement judiciaire d'exception (tant du point de vue de l'action éducative que des réponses pénales), et constituent en cela un symbole des discriminations institutionnelles que subissent, dans la société française, les populations Roms dans leur ensemble.

On pourrait arguer que ces « jeunes filles roumaines » sont à l'image des représentations médiatiques et politiques de la communauté Rom dans son ensemble : assimilées à une menace peu tangible mais fantasmée, à laquelle on ne donne ni parole, ni visage. Victimes d'une assignation raciale stigmatisante, la manière dont l'institution les prend en charge se traduit par un rejet symbolique hors du groupe des " filles », tant la distance établie dans l'institution judiciaire entre ces «jeunes filles roumaines » et «les filles»-c'est-à-dire toutes les autres -, est abyssale. Le fait de demander à une adolescente de 13 ans si elle a des enfants ou si elle s'est déjà prostituée (questions auxquelles ces «jeunes filles roumaines » doivent constamment répondre) les sépare radicalement du regard habituellement porté sur les jeunes filles domiciliées. Si ces « jeunes filles roumaines» ne sont pas tout à fait des jeunes filles « comme les autres », on pourrait à l'inverse, en forçant le trait et eu égard à la sur-pénalisation de leurs délits, considérer que le traitement qui leur est réservé en fait, au fond, « des garçons comme les autres »...

\section{Des adolescentes à l'intersection des rapports de pouvoir}

Les jeunes filles roumaines mettent en échec le système de genre habituellement observé dans la justice des mineurs. Leur situation invite le chercheur à affuter ses outils théoriques pour appréhender de nouvelles modalités de discrimination, encore mal renseignées. Différents rapports de pouvoir s'imbriquent dans l'exercice quotidien du droit et de la justice : les rapports de genre, de race, de classe se «co-constituent » et imprègnent les décisions de justice ${ }^{\mathrm{xi}}$. La sur-pénalisation des adolescentes roumaines doit interroger sans ménagement les impensés d'un système judiciaire relayant et produisant des discriminations conséquentes à l'égard de certains individus qui se trouvent, de plus, appartenir à une minorité ethno-raciale déjà fortement stigmatisée dans la société française. La reconnaissance des minorités et des discriminations spécifiques dont elles sont victimes semble se heurter, en France, à une idéologie républicaine $^{\mathrm{xii}}$ rendant invisibles ces discriminations quotidiennes et si difficiles à mesurer, y compris et surtout 
lorsqu'elles émergent au coeur des institutions de la République. Le cas des jeunes filles roumaines semble en mesure de fournir une critique par les faits de cette idéologie. Une approche intersectionnelle de la situation de ces adolescentes, favorisant l'analyse de la complexité des mécanismes discriminatoires au coeur d'un appareil judiciaire habituellement «bienveillant» à l'égard des adolescentes (sur le plan pénal, tout au moins), permet de pointer la banalité des discriminations au sein-même d'une institution censée mettre un terme à toute discrimination par un respect implacable de la règle de droit. S'il n'est pas certain qu'un droit anti-discriminatoire, encore peu développé au sein de la culture juridique française, suffirait à dépasser cette situation - à moins qu'il ne se fonde, précisément, sur une prise en compte fine des dominations imbriquées ${ }^{\text {xiii }}$-, il serait sans doute un premier pas vers une prise en compte des effets réels des discriminations de genre, race ou classe dans les arènes judiciaires les plus quotidiennes.

i

Cet article a notamment bénéficié des remarques et relectures de Marc Bessin, Coline Cardi, Meoïn Hagège et Sibylla Peron-Garvanoff, que je remercie chaleureusement.

ii Cet article est issu d'une thèse de doctorat en cours de rédaction, thèse réalisée sous la direction de Marc Bessin et Bertrand Pulman à l'Université Paris 13 (laboratoire IRIS) et portant sur les enjeux genrés de la justice des mineurs.

iii Principe central de l'Ordonnance du 2 février 1945, qui fixe les orientations de la justice pénale des mineurs.

iv On parle à cet égard de romaphobie. Cf. Plein droit, « Rom, n. et adj. : infra-étranger », décembre 2013.

$\mathrm{v}$ Les prénoms et noms des enquêtés ont été anonymisés.

vi Coline Cardi a largement travaillé ces dimensions en ce qui concerne la justice des majeurs. $C f$. Coline Cardi, « Le féminin maternel ou la question du traitement pénal des femmes », Pouvoirs, $\mathrm{n}^{\circ}$ 128, p. 75-86.

vii Le fait qu'il s'agisse d'une catégorie propre aux acteurs explique les guillemets systématiquement associés à cette expression dans ce texte.

viii Ethnographie basée sur deux mois d'observation, en complément d'une enquête déjà réalisée au Tribunal de Créteil, ainsi que sur des entretiens avec les éducateurs et l'observation de quelques entretiens éducatifs réalisés par les services de la PJJ au dépôt du tribunal de Paris.

ix D'après un responsable du service, les mineurs adhérant réellement à cette procédure représentent 40 adolescents sur les 200 annuellement déférés à Paris.

x La confusion fréquemment entretenue (par le Parquet notamment) entre le nombre de mineurs déférés différents et le volume total des défèrements amène parfois à sous-estimer le pourcentage de mineurs incarcérés.

xi Sirma Bilge, « De l'analogie à l'articulation : théoriser la différenciation sociale et l'inégalité complexe », L'homme et la société, vol. 2, n 176-177, 2010, p.59.

xii On lira avec intérêt l'ouvrage de Laurent Lévy, Le spectre du communautarisme, Paris, Éditions Amsterdam, 2005.

xiii Sirma Bilge et Olivier Roy, «La discrimination intersectionnelle : la naissance et le développement d'un concept et les paradoxes de sa mise en application en droit antidiscriminatoire », Canadian Journal of Law and Society, vol. $25, \mathrm{n}^{\circ} 1,2010$, p. 51-74. 